\title{
Action-Independent Subjective Expected Utility without States of the World
}

\author{
Andreas Duus Pape \\ Binghamton University Economics Department, Binghamton, USA \\ Email: apape@binghamton.edu
}

Received August 2, 2013; revised September 2, 2013; accepted September 10, 2013

Copyright (C) 2013 Andreas Duus Pape. This is an open access article distributed under the Creative Commons Attribution License, which permits unrestricted use, distribution, and reproduction in any medium, provided the original work is properly cited.

\begin{abstract}
This paper develops an axiomatic theory of decision-making under uncertainty that has no state-space. The choice setting follows Karni [1,2]: a set of effects (outcomes), a set of actions which induce these effects, and a set of real-valued bets over effects. In Karni's representation, a preference over action/bet pairs yields utility, which is action-dependent. In our representation, utility is action-independent. This is achieved by augmenting Karni's choice set with lotteries over actions. Identification is achieved similarly to Anscombe-Aumann [3], in which there are objective "roulette" lotteries over subjective "horse race" lotteries.
\end{abstract}

Keywords: Subjective Expected Utility; States of the World; Action Independence

\section{Introduction}

This paper develops an axiomatic theory of decisionmaking under uncertainty that has no state-space. The resulting representation has action-independent probabilities.

Karni $[1,2]$ proposes a decision theory without reference to states of the world. The primitives are effects (outcomes); actions, which induce these effects; and real-valued bets over which effect obtains. A preference relation over action/bet pairs yields a utility representation with subjective probability distributions over effects, one for each action. Karni's "main motivation" is to replace the state-space; he argues that "the relevant statespace is often unintuitive and too complex to be compatible with decision makers' perception of choice problems [1]". Our representation, like Karni, has actions, effects, bets, and no state-space. ${ }^{1}$

In Karni's representation, utility cannot be compared across actions; in our representation, it can. Establishing an action-independent representation in the Karni setting is the main motivation for our work. (Karni discusses action-independent preferences in his framework, but they do not emerge as a necessary consequence of a set of axioms.) Action-independence is similar to state-inde-

${ }^{1}$ Gilboa and Schmeidler [4] also develop a representation without a state-space. It differs from ours as it does from Karni; they take utility as given instead of deriving a utility function over bets and outcomes. pendence. As Karni points out [1], when his representation exhibits action-independence, "the probabilities ... represent the decision maker's beliefs in the sense of Ramsey [5]". Since our axioms deliver Karni's representation with action-independence, this applies to our representation (see Corollary 1).

The choice set is larger in our representation than that in Karni. Under Karni, the choice set is bets paired with actions, while in our representation, the choice set is bets paired with objective lotteries over actions. Ancombe-Aumann [3] augmented the Savage [6] choice set in a similar way. They added a layer of objective "roulette" bets on subjective "horse race" bets. Like AnscombeAumann, our representation requires objective lotteries ("roulette wheel" lotteries) to define the subjective probability distributions, which in the Anscombe-Aumann case are called "horse races", and here are the distributions over outcomes that result from actions.

Accordingly, like Anscombe-Aumann, the "essential device" of our representation "is to apply von NeumannMorgenstern's [7] utility theory twice over [3]". The proof here uses a similar process. We establish a utility representation over a subset of all actions: the "deterministic actions", which are actions that are known by the decision maker to achieve particular outcomes with certainty. Then we use the vNM axioms to extend this representation over the whole choice set.

Unlike Anscombe-Aumann, who used this technique 
to provide a representation, which was the same as the Savage representation, we provide for a representation which differs from the Karni representation in an important way: the application of this device in the Karni setting delivers an action-independent utility representation, which Karni's original framework does not deliver.

The remainder of our paper presents a set of axioms, a theorem with a corollary, and a proof. The theorem provides an equivalence between the axioms and an actionindependent representation without a state-space. The corollary states that our axioms imply an action-independent version of Karni, when attention is restricted to the Karni choice set.

\section{Notation and Framework}

$\Theta$, with typical element $\theta$, is a finite set of effects. $\mathbb{B}$ is the set of all bets $b: \Theta \rightarrow \mathbb{R}$. $\mathbb{A}$, with typical element $a$, is the possibly infinite set of actions. For any set $S$, define $\mathcal{L}(S)$ as the set all of simple lotteries over $S . \succ$ is a binary relation defined on the choice set $\mathbb{C}$, where $\mathbb{C}=\mathcal{L}(\mathbb{A}) \times \mathbb{B}$, with typical element $(\alpha, b), \quad \alpha \in \mathcal{L}(\mathbb{A}), b \in \mathbb{B} . \alpha(a)$ will be understood to be the probability that the action $a$ occurs under lottery $a$ and $\mathbb{A}(\alpha)$ will be understood to be the (finite) subset of $\mathbb{A}$ upon which $a$ puts positive probability. For notational simplicity, we will also interpret $a \in \mathbb{A}$ to mean the degenerate lottery over actions, which yields action $a$ with certainty. We define a mixture of elements of $\mathbb{C}$ element-wise:

$$
\begin{aligned}
& \beta(\alpha, b)+(1-\beta)\left(\alpha^{\prime}, b^{\prime}\right) \\
& =\left(\beta \alpha+(1-\beta) \alpha^{\prime}, \beta b+(1-\beta) b^{\prime}\right) \in \mathbb{C}
\end{aligned}
$$

where $\beta$ is some objective probability, and where $\beta \alpha+(1-\beta) \alpha^{\prime}$ and $\beta b+(1-\beta) b^{\prime}$ are also defined element-wise. Definition of "null": an effect $\theta$ is null given the action $\mathrm{a}$ if $(a, b) \sim\left(a, b^{\prime}\right)$ for all bets $b, b^{\prime}$ such that $b(\theta) \neq b^{\prime}(\theta)$ and $b\left(\theta^{\prime}\right)=b^{\prime}\left(\theta^{\prime}\right)$ for all $\theta^{\prime} \neq \theta$. The definitions of $\Theta, \mathbb{B}, \mathbb{A}$, and "null given action $a "$ follow Karni [1].

A deterministic action $a_{\theta}$ is an action which yields the effect $\theta$ with certainty, in the sense that: given action $a_{\theta}$, all $\theta^{\prime} \neq \theta$ are null and $\theta$ is not null. We also assume that there is no more than one deterministic action for each outcome. Let $\mathbb{A}_{\Theta}$ be the set of all deterministic actions. We suppose that $\mathbb{A}_{\Theta} \subseteq \mathbb{A}$, i.e. for all $\theta \in \Theta, a_{\theta} \in \mathbb{A}$. This assumption is critical for our representation; it is similar to Karni's assumption A0 in that it generates sufficient richness of the choice set.

An example: $\Theta=\{$ Dry, Wet $\}, \mathbb{A}=\{$ Stay in Room; Walk without Umbrella, Walk with Umbrella; Take a Shower $\}$, and $\mathbb{A}_{\Theta}=$ \{Stay in Room; Take a Shower under the assumption that Stay in Room determines the effect Dry and that Take a Shower determines the effect Wet.

\section{Axioms}

Axioms 1 through 3 are standard von Neuman-Morgenstern axioms over objects in $\mathbb{C}$. We will refer to them collectively as "the vNM axioms".

Axiom 1. (Preference Relation.) $\succ$ on $\mathbb{C}$ is asymetric and negatively transitive.

Axiom 2. (Independence.) Suppose $(\alpha, b),\left(\alpha^{\prime}, b^{\prime}\right)$, $\left(\alpha^{\prime \prime}, b^{\prime \prime}\right) \in \mathbb{C}$. Then $(\alpha, b) \succ\left(\alpha^{\prime}, b^{\prime}\right)$ if and only if

$$
\begin{aligned}
& \beta(\alpha, b)+(1-\beta)\left(\alpha^{\prime \prime}, b^{\prime \prime}\right) \\
& \succ \beta\left(\alpha^{\prime}, b^{\prime}\right)+(1-\beta)\left(\alpha^{\prime \prime}, b^{\prime \prime}\right) \text { for any } \beta \in(0,1]
\end{aligned}
$$

Axiom 3. (Continuity.) Suppose $(\alpha, b),\left(\alpha^{\prime}, b^{\prime}\right)$, $\left(\alpha^{\prime \prime}, b^{\prime \prime}\right) \in \mathbb{C}$, where $(\alpha, b) \succ\left(\alpha^{\prime}, b^{\prime}\right) \succ\left(\alpha^{\prime \prime}, b^{\prime \prime}\right)$. Then there exist $\beta, \gamma \in(0,1)$ such that

$$
\begin{aligned}
& \beta(\alpha, b)+(1-\beta)\left(\alpha^{\prime \prime}, b^{\prime \prime}\right) \\
& \succ\left(\alpha^{\prime}, b^{\prime}\right) \succ \gamma(\alpha, b)+(1-\gamma)\left(\alpha^{\prime \prime}, b^{\prime \prime}\right)
\end{aligned}
$$

Axiom 4. (Bet monotonicity.) Let $b, b^{\prime} \in \mathbb{B}$ such that $b(\theta)>b^{\prime}(\theta)$ for some $\theta \in \Theta$ and $b\left(\theta^{\prime}\right)=b^{\prime}\left(\theta^{\prime}\right)$ for all $\theta^{\prime} \in \Theta, \theta^{\prime} \neq \theta$. Then, for all $\alpha \in \mathcal{L}\left(\mathbb{A}_{\Theta}\right)$ such that $\alpha\left(a_{\theta}\right)>0,(\alpha, b) \succ\left(\alpha, b^{\prime}\right)$.

Axiom 4 considers two bets, $b$ and $b^{\prime}$, which are identical but for one effect, $\theta$. On effect $\theta$, bet $b$ yields a higher payoff than $b^{\prime}$. Then it considers lotteries over deterministic actions which yield effect $\theta$ with positive objective probability. Each of those lotteries, paired with bet $b$, must be preferred to the same lottery paired with bet $b^{\prime}$. The logic is, the only difference between those lottery/bet pairs is what bet payoff is received when effect $\theta$ occurs, and in that case, the agent should prefer more wealth to less. This axiom, then, codifies that more wealth is better. Importantly, this axiom also delivers that association of effects with bets which pay off when that effect occurs. That is, it assures that, when the decision maker is considering the choice object $\left(a_{\theta}, b\right)$, the payoff given by bet $b$ under some other effect $\theta^{\prime} \neq \theta$ is irrelevant.

Axiom 5. Suppose $a \in \mathbb{A}$. Then there exists $\alpha \in \mathcal{L}\left(\mathbb{A}_{\Theta}\right)$ such that

$$
(a, b) \sim(\alpha, b) \quad \forall b \in \mathbb{B}
$$

This axiom encodes the idea that an action is of interest to the decision maker only through the bouquet of effects it delivers by requiring that each action is in essence identical to some lottery over deterministic actions. In our example with $\Theta=\{$ Dry, Wet $\}$, it would require that action Walk with Umbrella be indifferent for all bets to some lottery over Stay in Room and Take a Shower.

From this perspective, this axiom can be thought of as a lottery-reduction axiom: that the decision maker is only

${ }^{2}$ This formulation of the vNM axioms follows Kreps [8], p. 43-44, with the corresponding vNM theorem for an arbitrary prize space and simple lotteries given by Theorem 5.15, p. 58 . 
ultimately concerned about the outcomes and not the means by which those outcomes are achieved.

The lottery $\alpha$ which corresponds to action $a$ is unique for each action (shown below, Section 4, step three). Given this fact, the weight $a$ puts on deterministic action $a_{\theta}$ will be interpreted as the probability the agent believes the action $a$ induces effect $\theta$.

This interpretation might raise an objection from some readers, which we address here. Consider a lottery over deterministic actions, which yields outcome $\theta$ which probability $p$ and outcome $\theta^{\prime}$ with probability $1-p$ : $i . e$. the lottery $p a_{\theta}+(1-p) a_{\theta^{\prime}}$. Now suppose the action $a$ also yields the same outcomes $\theta$ and $\theta^{\prime}$ with the same subjective probabilities $p$ and $1-p$ : In the identification of the representation below, Axiom 5 is used in such a way that, it must be the case that

$a \sim p a_{\theta}+(1-p) a_{\theta^{\prime}}$, else the representation yields the incorrect subjective probabilities.

The reader may object to this interpretation for the following reason: it could be the case that the lottery is strictly preferred to action $a$. Why? This would be the case, it could be argued, if action $a$ is associated with some cost that the lottery over deterministic actions is not subject to. Therefore, the argument goes, interpreting this axiom in this way, and using it to specify subjective probabilities, is equivalent to assuming that all actions are costless (or, more generally, that they have the same cost.)

The conclusion of this objection, however, is unwarranted, which can be seen when one considers the set of outcomes $\Theta . \Theta$ is an arbitrary set. Suppose that the elements of $\Theta$ are final levels of wealth (excluding wealth from the bets), so that $\Theta=R$. Seen from this perspective, it is possible to imagine two actions that have different costs: Consider, as before, an action $a$ which yields the outcome $\theta$ with subjective probability $p$ and the outcome $\theta^{\prime}$ with subjective probability $\theta^{\prime}$ : Now consider action $a^{\prime}$ which yields the outcomes $(\theta-c)$ with subjective probability $p$ and outcome $\left(\theta^{\prime}-c\right)$ with subjective probability $1-p$, for some $c>$ 0 : It could be said that action $a^{\prime}$ is identical to action $a^{\prime}$; but for a cost $c$. This examples reveals that this frame- work does allow for actions with different costs; those costs must be expressed, however, in terms of final outcomes. It does not allow for costs that are not observable to the modeler.

\section{A Representation Theorem}

As per the standard definition, we say $U(\alpha, b)$ represents $\succ$ if

$$
U(\alpha, b)>U\left(\alpha^{\prime}, b^{\prime}\right) \Leftrightarrow(\alpha, b) \succ\left(\alpha^{\prime}, b^{\prime}\right) \forall(\alpha, b) \in \mathbb{C} \quad .
$$
Then:

Theorem 1. The following two statements are equivalent:
1) $\succ$ satisfies Axioms 1-5;

2) There exists $u: \Theta \times \mathbb{B} \rightarrow \mathbb{R}$ unique up to a positive affine transformation and unique $\pi: \mathbb{A} \rightarrow \mathcal{L}(\Theta)$ such that $U(\alpha, b)$ represents $\succ$, where

$$
U(\alpha, b)=\sum_{a \in \mathbb{A}(\alpha)} \alpha(a)\left[\sum_{\theta \in \Theta}(u(\theta, b(\theta)) \pi(\theta \mid a))\right] .
$$

Corollary 1. If $\succ$ satisfies Axioms 1 - 5, then on $\mathbb{C}_{K}=\{(a, b) \mid a \in \mathbb{A}, b \in \mathbb{B}\}, \succ$ can be represented by:

$$
U_{\text {Karni }}(a, b)=f_{a}\left(\sum_{\theta \in \Theta} u(\theta, b(\theta)) \pi(\theta \mid a)\right)
$$

for some $u: \Theta \times \mathbb{B} \rightarrow \mathbb{R}$ unique up to a positive affine transformation, and unique $\pi: \mathbb{A} \rightarrow \mathcal{L}(\Theta)$, and for all $a \in \mathbb{A}, f_{a}$ is the identity function.

Corollary 1 is written in this way to emphasize that $U_{\text {Karni }}(a, b)$ is the representation given in Karni, save that the functions $f_{a}$ in Karni may vary across actions. Hence our representation implies an action-independent version of Karni's representation [1].

Proof. The corollary is an immediate implication of theorem 1 and requires no proof. The proof that the representation implies the axioms (i.e. statement 2 implies statement 1) is straightforward and is omitted. What follows is the proof that the axioms imply the representation. The proof proceeds in four steps.

First, we establish a von Neumann-Morgenstern representation on lotteries over deterministic actions paired with bets.

Consider the preferences $\succ$ on the set $\mathbb{C}_{\Theta}=$ $\mathcal{L}\left(\mathbb{A}_{\Theta}\right) \times \mathbb{B}$. The vNM axioms and the vNM theorem imply that there exists $\hat{u}()$, unique up to a positive, affine transformation, such that $\hat{\mathbb{U}}(\alpha, b)$ represents $\succ$ on $\mathbb{C}_{\Theta}$, where $\hat{\mathbb{U}}(\alpha, b)=\sum_{\theta \in \Theta} \alpha\left(a_{\theta}\right) \hat{u}\left(a_{\theta}, b\right)$. Now define $\breve{u}(\theta, b)=\hat{u}\left(a_{\theta}, b\right)$. This is well-defined because we assume that every outcome has exactly one corresponding deterministic action and vice versa. Then we can equivalently define $\hat{\mathbb{U}}()$ as:

$$
\hat{\mathbb{U}}(\alpha, b)=\sum_{\theta \in \Theta} \alpha\left(a_{\theta}\right) \breve{u}(\theta, b) .
$$

Second, we establish that $\breve{u}(\theta, b)$ does not depend on all values in the vector $b$; instead, it only depends on the value of $b(\theta)$. Then, we introduce a representation which takes advantage of this fact.

Suppose $b, b^{\prime} \in \mathbb{B}$ such that $b(\theta)=b^{\prime}(\theta)$. Let $\alpha_{\theta}$ be the lottery in $\mathcal{L}\left(\mathbb{A}_{\theta}\right)$ which puts all weight on action $\alpha_{\theta}$. Note that $\left(\alpha_{\theta}, b\right) \sim\left(\alpha_{\theta}, b^{\prime}\right)$ because bets $b$ and $b^{\prime}$ differ only on events that are null given action $\alpha_{\theta}$.

$$
\begin{aligned}
\left(\alpha_{\theta}, b\right) \sim\left(\alpha_{\theta}, b^{\prime}\right) & \Rightarrow \hat{\mathbb{U}}\left(\alpha_{\theta}, b\right)=\hat{\mathbb{U}}\left(\alpha_{\theta}, b^{\prime}\right) \\
& \Rightarrow \breve{u}(\theta, b)=\breve{u}\left(\theta, b^{\prime}\right)
\end{aligned}
$$

Define $u(\theta, r), \quad r \in \mathbb{R} \quad$ as: $u(\theta, r)=\breve{u}\left(\theta, b_{r}\right)$ where $b_{r}$ is a bet that yields $r$ for every effect. Define 
$\mathbb{U}(\cdot)$ as:

$$
\mathbb{U}(\alpha, b)=\sum_{\theta \in \Theta} \alpha\left(a_{\theta}\right) u(\theta, b(\theta)) .
$$

Given that only the $\theta^{\text {th }}$ element of $b$ matters, that $\hat{\mathbb{U}}(\cdot)$ represents $\succ$ on $\mathbb{C}_{\Theta}$ implies $\hat{\mathbb{U}}(\cdot)$ also represents $\succ$ on $\mathbb{C}_{\Theta}$.

Third: By Axiom 5, each action is indifferent to at least one lottery over deterministic actions. Here we establish that each action is indifferent to no more than one such lottery.

Let $a \in \mathbb{A}$ and $\alpha \in \mathcal{L}\left(\mathbb{A}_{\Theta}\right)$, such that $\alpha$ is indifferent to a for all bets, in the sense of Axiom 5. Suppose toward a contradiction that there exists $\alpha^{\prime} \in \mathcal{L}\left(\mathbb{A}_{\Theta}\right)$, such that $\alpha \neq \alpha^{\prime}$ and $\alpha^{\prime}$ is indifferent to a for all bets as in the sense of Axiom 5. Then, $\alpha^{\prime}$ is indifferent to $\alpha$ for all bets as in the sense of Axiom 5.

$\alpha^{\prime} \neq \alpha$, and that they are distributions, implies that, for at least one $\theta \in \Theta, \alpha(\theta)>\alpha^{\prime}(\theta)$. For notational simplicity suppose that $\theta$ has index 1 . Then interpret $\alpha$ and $\alpha^{\prime}$ as $|\Theta|$-dimensional vectors and express them as:

$$
\begin{gathered}
\alpha=(1-\alpha(\theta))\left(0, \frac{\alpha\left(\theta_{2}\right)}{1-\alpha(\theta)}, \cdots, \frac{\alpha\left(\theta_{|\Theta|}\right)}{1-\alpha(\theta)}\right)^{\mathrm{T}} \\
+\left(\alpha(\theta)-\alpha^{\prime}(\theta)\right)(1,0, \cdots, 0)^{\mathrm{T}}+\alpha^{\prime}(\theta)(1,0, \cdots, 0)^{\mathrm{T}} \\
\alpha^{\prime}=\left(1-\alpha^{\prime}(\theta)\right)\left(0, \frac{\alpha^{\prime}\left(\theta_{2}\right)}{1-\alpha^{\prime}(\theta)}, \cdots, \frac{\alpha^{\prime}\left(\theta_{|\Theta|}\right)}{1-\alpha^{\prime}(\theta)}\right)^{\mathrm{T}} \\
+\alpha^{\prime}(\theta)(1,0, \cdots, 0)^{\mathrm{T}}
\end{gathered}
$$

Since $\alpha \sim \alpha^{\prime}$ for all bets, then, by the independence axiom, the following two lotteries, $\tilde{\alpha}$ and $\tilde{\alpha}^{\prime}$, are also indifferent for all bets:

$$
\begin{gathered}
\tilde{\alpha}=\frac{1-\alpha(\theta)}{1-\alpha^{\prime}(\theta)}\left(0, \frac{\alpha\left(\theta_{2}\right)}{1-\alpha(\theta)}, \cdots, \frac{\alpha\left(\theta_{|\Theta|}\right)}{1-\alpha(\theta)}\right)^{\mathrm{T}} \\
+\frac{\alpha(\theta)-\alpha^{\prime}(\theta)}{1-\alpha^{\prime}(\theta)}(1,0, \cdots, 0)^{\mathrm{T}} \\
\tilde{\alpha}^{\prime}=\left(0, \frac{\alpha^{\prime}\left(\theta_{2}\right)}{1-\alpha^{\prime}(\theta)}, \cdots, \frac{\alpha^{\prime}\left(\theta_{|\Theta|}\right)}{1-\alpha^{\prime}(\theta)}\right)^{\mathrm{T}}
\end{gathered}
$$

Now consider some bet $b$ and some bet $b^{\prime}$, where $b^{\prime}\left(\theta^{\prime}\right)=b\left(\theta^{\prime}\right)$ for all $\theta^{\prime} \neq \theta, \theta^{\prime} \in \Theta$, but $b^{\prime}(\theta)>b(\theta)$. By independence (axiom 2), $\left(\tilde{\alpha}^{\prime}, b\right) \sim\left(\tilde{\alpha}^{\prime}, b^{\prime}\right)$ since the action $a_{\theta}$ occurs with probability zero under $\tilde{\alpha}^{\prime}$. But $\left(\tilde{\alpha}, b^{\prime}\right) \succ(\tilde{\alpha}, b)$ by bet monotonicity (Axiom 4). This implies that $\tilde{\alpha}$ and $\tilde{\alpha}^{\prime}$ are not indifferent for at least one bet of $b$; $b 0$ : Contradiction.
Fourth, we interpret the unique corresponding lottery over deterministic actions as a subjective probability distribution, and apply vNM to the entire choice set $\mathbb{C}$.

For each $a$, let $\alpha_{a}$ be the unique deterministic action lottery $\alpha_{a} \in \mathcal{L}\left(\mathbb{A}_{\Theta}\right)$ such that $\alpha_{a}$ is indifferent to a for all bets in the sense of Axiom 5. Define the family of probability distributions $\pi$ as: $\pi(\theta \mid a)=\alpha_{a}\left(a_{\theta}\right)$.

Consider some $\alpha \in \mathcal{L}(\mathbb{A})$. By the vNM axioms and the vNM theorem, $U(\alpha, b)=\sum_{a \in \mathbb{A}(\alpha)} v(a, b) \alpha(a)$

represents $\succ$ on $\mathbb{C}$, where $v(a, b)$ is unique up to a positive affine transformation. Now consider an arbitrary degenerate lottery $a$ and bet $b . U(a, b)=v(a, b)$ and $\mathbb{U}\left(\alpha_{a}, b\right)=\sum u(\theta, b(\theta)) \pi(\theta \mid a)$. Since $a$ and $b$ were chosen arbitrarily, this implies $v(a, b)$ can be affinetransformed into $\tilde{v}$ such that

$\tilde{v}(a, b)=\sum u(\theta, b(\theta)) \pi(\theta \mid a) \quad$ for $\quad$ all $\quad a \in \mathbb{A}, b \in \mathbb{B}$. Therefore the following function represents $\succ$ on $\mathbb{C}$ :

$$
U(\alpha, b)=\sum_{a \in \mathbb{A}(\alpha)} \alpha(a)\left[\sum_{\theta \in \Theta}(u(\theta, b(\theta)) \pi(\theta \mid a))\right]
$$

where the $\pi$ are unique and $u$ is unique up to a positive affine transformation.

\section{Conclusion}

This paper develops an axiomatic theory of decisionmaking under uncertainty that has no state-space. The resulting representation has action-independent probabilities. The primitives are effects (outcomes); actions, which induce these effects; and real-valued bets over which effect obtains. This representation is most closely related to Karni [1,2], which also proposes a decision theory without reference to states of the world. In Karni's representation, unlike ours, however, the probability distributions are action dependent, which results in probabilities that can not be compared across actions. In our representation, the probability distributions are action independent, so they can be compared across actions and therefore are probabilities in the sense of Ramsey [5].

\section{REFERENCES}

[1] E. Karni, "Subjective Expected Utility Theory without States of the World," Journal of Mathematical Economics, Vol. 42, No. 3, 2006, pp. 325-342.

http://dx.doi.org/10.1016/j.jmateco.2005.08.007

[2] E. Karni, "A Theory of Bayesian Decision Making with Action-Dependent Subjective Probabilities," Economic Theory, Vol. 48, No. 1, 2011, pp. 125-146.

[3] F. Anscombe and R. Aumann, "A Definition of Subjective Probability," The Annals of Mathematical Statistics, Vol. 34, No. 1, 1963, pp. 199-205.

[4] I. Gilboa and D. Schmeidler, "Subjective Distributions," Theory and Decision, Vol. 56, No. 4, 2004, pp. 345-357. 
http://dx.doi.org/10.1007/s11238-004-2596-7

[5] F. P. Ramsey, "The Foundations of Mathematics and Other Logical Essays: Truth and Probability," Routledge and Kegan Paul, London, 1931, pp. 156- 198.

[6] L. J. Savage, "The Foundations of Statistics," Wiley, Hoboken, 1954.
[7] J. von Neumann and O. Morgenstern, "Theory of Games and Economic Behavior," Princeton University Press, Princeton, 1944.

[8] D. M. Kreps, "Notes on the Theory of Choice. Underground Classics in Economics," Westview Press, Inc., Fredrick A. Praeger, 1988. 